\title{
On the use of low-pass filters in high-gain observers th
}

\author{
Daniele Astolfia ${ }^{\mathrm{a}}$ Luca Zaccarian $^{\mathrm{b}}$, Marc Jungers $^{\mathrm{c}}$ \\ ${ }^{a}$ Univ Lyon, Université Claude Bernard Lyon 1, CNRS, LAGEPP UMR 5007, 43 boulevard du 11 novembre 1918, F-69100, Villeurbanne, France. \\ ${ }^{b}$ LAAS-CNRS, Université de Toulouse, CNRS, Toulouse, France and Dept. of Industrial Engineering, University of Trento, Italy. \\ ${ }^{c}$ Université de Lorraine, CNRS, CRAN, F-54000 Nancy, France
}

\begin{abstract}
To address the well-known noise sensitivity problems associated with high-gain observers, we insert a low-pass filter on the measurement channel. Considering nonlinear plants in observability canonical form, we first motivate an architecture where the output error is filtered by a linear system parametrized by its arbitrary order and a scalar positive gain. Our main result establishes an exponential finite gain bound for the estimation error, from the measurement noise, this gain being dependent on the high-gain and filter parameters. We also prove bounds depending on the filter parameters characterizing improved high-frequency gains from the measurement noise to the estimation error. The proposed construction is shown to behave desirably in numerical simulations.
\end{abstract}

Keywords: High gain observers, Low-pass filters, Noise analysis

\section{Introduction}

High-gain observers appeared in the literature at the beginning of the 90's, see [19] and references therein for an historical survey. This class of observers is a popular tool for state estimation of nonlinear dynamical systems, due to the remarkable feature of having a convergence rate that can be arbitrarily tuned by adjusting only one single "high-gain" parameter. As showed in [8], this property is fundamental for establishing a nonlinear separation principle in output feedback stabilization of nonlinear systems. Nevertheless, due to their high sensitivity to measurement noise, high-gain observers are rarely applied in practice. Performance improvement in the presence of noise is therefore one of the main motivations that pushed the last years of research towards alternative designs preserving the good features of high-gain observers, namely fast convergence and good robustness properties with respect to model uncertainties, while mitigating the effect of (high-frequency) measurement noise at the steady state. In this context, it is fundamental to recall that the performance of a high-gain observer in the presence of colored measurement noise is mainly characterized by its dominant linear behavior, if the frequency of the noise is large enough, as showed in [5], [20]. In particular, the role of the relative degree between the measurement and the state estimate plays a fundamental role, and higher relative degrees allows improving the high-frequency filtering properties. This crucial observation has given a new impulse to the research on high-gain observers, inspiring a number of solutions aimed at augmenting this relative degree while preserving other features

\footnotetext{
This work was supported in part by the Agence nationale de la recherche (ANR) through Project HANDY under Grant ANR-18-CE40-0010.

Email addresses: daniele.astolfi@univ-lyon1.fr (Daniele Astolfi), zaccarian@laas.fr (Luca Zaccarian), marc.jungers@univ-lorraine.fr (Marc Jungers)
}

of the estimates, see $[3,4,7,6,18,20,26,27,28]$. The same idea of using a filtered version of the output has been also proposed in the design of Proportional Integral (PI) observers, see $[9,10]$. Therein, it is shown how to design an observer that combines both the measurement and its filtered version. The same idea has also been recently re-proposed, in the context of linear observers, with the use of an arbitrary order of integral actions, i.e. a $\mathrm{PI}^{\rho}$ observer, see [24], although the extension to a nonlinear framework is not straightforward. In the context of sliding-mode observers, the same idea of pre-filtering the output injection term has been recently proposed in [23]. Finally, alternative designs, making use of saturated and deadzonated output injections with adaptive thresholds have been proposed in $[1,13,2]$.

In line with those works, we propose here a simple but effective solution that aims at increasing the relative degree between the measurement and the observer, while maintaining its fundamental properties. In particular, the output injection term is filtered by a low-pass filter of any desired order and re-injected into the observer dynamics. This design is similar to [20] and [28] but exhibits some fundamental differences. Differently from [20], asymptotic convergence of the estimation error is still preserved in the absence of measurement noise. Furthermore, differently from [28], the proposed design is simpler as only one single parameter needs to be tuned. Furthermore, the design presents a useful degree of freedom as the dimension of the filter can be arbitrarily chosen. The proposed structure is a simple "plug-and-play" tool that can be used in the design of high-gain observers, which is simpler than the constructions proposed in $[6,18,26]$. Finally, differently from [9], we do not need here to duplicate the filter in the observer design.

The rest of the paper is organized as follows. The main results on high-gain observers are highligted in Section 2. We explain the new proposed structure in Section 3 and we provide 
the main result analyzing the convergence properties of the new observer in Section 4. The effect of high-frequency measurement noise is discussed in Section 5. A numerical example is given in Section 6. Conclusions are derived in Section 7.

Notation. We denote with $\mathbb{R}$ the set of real numbers, with $\mathbb{N}$ the set of non-negative integers and $\mathbb{N}_{>0}=\{1,2, \ldots\}$. A vector $x=\left[\begin{array}{lll}x_{1} & \cdots & x_{n}\end{array}\right]^{\top} \in \mathbb{R}^{n}$ is often denote by $x=\operatorname{col}\left(x_{1}, \ldots, x_{n}\right)$ or simply $x=\left(x_{1}, \ldots, x_{n}\right)^{\top}$. We denote with $|\cdot|$ the standard Euclidean norm, and with $\|s\|_{\infty}=\sup _{t \in[0, \infty)}|s(t)|$. Given a square matrix $F$, we denote $p_{F}(\lambda)$ its characteristic polynomial, namely $p_{F}(\lambda)=\operatorname{det}(\lambda I-F)$ and $\sigma(F)$ its spectrum. For a symmetric matrix $P, \lambda_{\max }(P)$ is its maximal eigenvalue. We denote with $I_{i}$, or simply $I$, an identity matrix of dimension $i \in \mathbb{N}_{>0}$. We denote with $0_{i, j}$ a zero matrix of dimension $i \times j$, with $i, j \in \mathbb{N}_{>0}$. We denote with $\mathbb{1}_{i}$ the all-ones $i$-vector $\mathbb{1}_{i}=\operatorname{col}(1,1, \ldots, 1)$. We denote a triplet in prime form $\left(A_{i}, B_{i}, C_{i}\right)$ of dimension $i \in \mathbb{N}_{>0}$, (or simply $(A, B, C)$ in the case of no dimension ambiguity), matrices of the form

$$
A_{i}:=\left(\begin{array}{cc}
0_{i-1,1} & I_{i-1} \\
0 & 0_{1, i-1}
\end{array}\right), \quad B_{i}:=\left(\begin{array}{c}
0_{i-1,1} \\
1
\end{array}\right), \quad C_{i}:=\left(\begin{array}{ll}
1 & 0_{1, i-1}
\end{array}\right) .
$$

Finally, given $\ell \in \mathbb{N}_{>0}$, we define $D_{n}(\ell)$ as the diagonal matrix containing increasing powers of $\ell$, that is,

$$
D_{n}(\ell):=\operatorname{diag}\left(\ell, \ell^{2}, \ldots, \ell^{n}\right) .
$$

\section{Background on High-Gain Observers}

Consider a system in the observability canonical form ${ }^{1}[14]$

$$
\begin{aligned}
\dot{x}_{i} & =x_{i+1}, \quad i=1, \ldots, n-1, \\
\dot{x}_{n} & =\varphi(x, u), \\
y & =x_{1}+v
\end{aligned}
$$

equivalently written in the compact form

$$
\dot{x}=A_{n} x+B_{n} \varphi(x, u), \quad y=C_{n} x+v,
$$

where $x=\left(x_{1}, \ldots, x_{n}\right)^{\top} \in \mathbb{R}^{n}$ is the state, $u \in \mathbb{R}^{m}$ is a known input, $y \in \mathbb{R}$ is a scalar measured output, $\left(A_{n}, B_{n}, C_{n}\right)$ is a triplet in prime form of dimension $n$, see (1), and $v: \mathbb{R}_{\geq 0} \rightarrow \mathbb{R}$ is some (bounded) measurement noise. In this work, we address the observer design problem for system (3) in a semi-global scenario, namely we suppose that function $\varphi$ is locally Lipschitz and that there exist compact sets $\mathcal{U} \subset \mathbb{R}^{m}$ and $\mathcal{X}_{0}, \mathcal{X}$ satisfying $\mathcal{X}_{0} \subseteq \mathcal{X} \subset \mathbb{R}^{n}$, such that, for any initial condition $x(0) \in \mathcal{X}_{0}$ and under the action of any control input $u: \mathbb{R}_{\geq 0} \rightarrow \mathcal{U}$, the solution of (3) remains in $\mathcal{X}$ for all $t \geq 0$. When $\varphi$ is globally Lipschitz, then we can allow $\mathcal{X}=\mathbb{R}^{n}$.

A high-gain observer for system (3) can be therefore designed as

$$
\dot{\hat{x}}=A_{n} \hat{x}+B_{n} \varphi_{s}(\hat{x}, u)+D_{n}(\ell) K_{n} \eta,
$$

\footnotetext{
${ }^{1}$ The results presented in this work can be easily extended to more general systems where each component $x_{i}$ is governed by dynamics of the form $\dot{x}_{i}=$ $x_{i+1}+\varphi_{i}\left(x_{1}, \ldots, x_{i}, u\right)$ for all $i=1, \ldots, n-1$.
}

where $\hat{x}=\left(\hat{x}_{1}, \ldots, \hat{x}_{n}\right)^{\top} \in \mathbb{R}^{n}$ is the state of the observer with initial conditions $\hat{x}(0) \in \mathbb{R}^{n}, \eta \in \mathbb{R}$ is the correction term defined as

$$
\eta=y-\hat{x}_{1},
$$

$D_{n}(\ell)$ is defined as in (2), with $\ell \geq 1$ being the so-called high-gain parameter, matrices $A_{r}, B_{r}$ and $C_{r}$ are defined in (1), $K_{n}:=\left(k_{1}, \ldots, k_{n}\right)^{\top}$ is a vector of gains chosen so that the matrix $\left(A_{n}-K_{n} C_{n}\right)$ is Hurwitz, and $\varphi_{s}$ is a globally bounded and locally Lipschitz function satisfying

$$
\left|\varphi(x, u)-\varphi_{s}(\hat{x}, u)\right| \leq \bar{\varphi}|x-\hat{x}|
$$

for all $x \in \mathcal{X}, \hat{x} \in \mathbb{R}^{n}, u \in \mathcal{U}$, and for some scalar $\bar{\varphi}>0$. For instance, $\varphi_{s}$ can be selected by saturating (component-wise or globally) the function $\varphi$ outside the compact sets $\mathcal{X}, \mathcal{U}$. When the function $\varphi$ is globally Lipschitz, then the function $\varphi_{s}$ can be selected equal to $\varphi$ on all of its domain, thus relaxing the global boundedness requirement. The subscript $s$ resembles the fact that a saturated version of function $\varphi$ outside the compact sets $\mathcal{X}$ and $\mathcal{U}$ is an adequate choice.

The high-gain observer (4)-(5) can be represented by a block diagram scheme as in Figure 1. When $\ell$ is chosen large enough, the following bound holds for each $i=1, \ldots, n$, see [19],

$$
\left|x_{i}(t)-\hat{x}_{i}(t)\right| \leq \alpha \ell^{i-1} \exp (-\beta \ell t)|x(0)-\hat{x}(0)|+\gamma \ell^{i-1}\|v\|_{\infty}
$$

for all $t \in \mathbb{R}_{\geq 0}$, for all initial conditions $(x(0), \hat{x}(0)) \in \mathcal{X}_{0} \times \mathbb{R}^{n}$ and for some $\alpha, \beta, \gamma>0$ independent of $\ell$. Inequality (7) shows that the estimation error converges exponentially to zero with an arbitrarily fast convergence rate that can be tuned by increasing the high-gain parameter $\ell$. In addition to this, the estimation error satisfies an input-to-state stability property with respect to the measurement noise $v$, with a gain proportional to $\ell^{n-1}$, namely the faster the exponential rate of the observer, the worse the asymptotic behavior in the presence of measurement noise. In [5] it has also been shown that the low-pass filtering behavior of the high-gain observer (4)-(5) can be established by modeling the measurement noise as a finite sum of sinusoids, namely

$$
v(t):=\sum_{i=1}^{N} v_{i} \sin \left(\frac{\omega_{i}}{\varepsilon} t+\phi_{i}\right),
$$

for some $N>0$, where parameter $\varepsilon>0$ shifts towards infinity the basic frequencies $\omega_{i}$ as it approaches zero, while $v_{i}>0$ and $\phi_{i}$ characterize the amplitude and the phase of each component, respectively. The measurement noise (8) has a high-frequency behavior for $\varepsilon$ small enough and the asymptotic behavior of the high-gain observer can be characterized as

$$
\limsup _{t \rightarrow+\infty}\left|x_{i}(t)-\hat{x}_{i}(t)\right| \leq \varepsilon \varrho \ell^{i}\|v\|_{\infty}
$$

where scalar $\varrho>0$ is independent of $\ell$. It is readily seen that bound (9) captures the low-pass filtering behaviour of the highgain observer (4)-(5) since $\lim _{\mathcal{E} \rightarrow 0} \lim _{t \rightarrow+\infty}|x(t)-\hat{x}(t)|=0$. On the other hand, bound (9) highlights that the asymptotic gain at high-frequencies is proportional to $\ell^{i}$ and not to $\ell^{i-1}$, as one may expect from (7). 


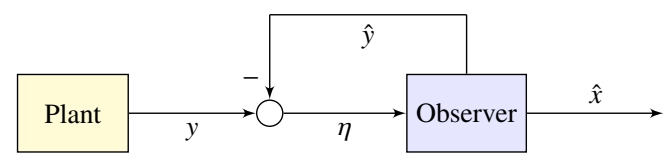

Figure 1: Standard observer scheme.

\section{Proposed architecture}

In this work, we investigate the use of low-pass linear filters to improve the sensitivity properties (9) of the high-gain observer (4) with respect to high frequency measurement noise, without losing its desirable properties (7) in terms of asymptotic and arbitrarily fast convergence rate and input-to-state stability with respect to $v$. The main idea that we pursue is to augment the relative degree between the measured output $y$ and the state estimate $\hat{x}$, in order to improve the asymptotic bound (9). To this end, we replace the static correction term (5) with the following filtered version

$$
\begin{aligned}
\dot{z}_{1} & =-\frac{\ell}{\theta}\left(z_{1}-\left(y-\hat{x}_{1}\right)\right), \\
\dot{z}_{i} & =-\frac{\ell}{\theta}\left(z_{i}-z_{i-1}\right), \quad i=2, \ldots, r, \\
\eta & =z_{r} .
\end{aligned}
$$

As shown in Figure 3, this corresponds to adding a pool of $r$ identical first-order strictly proper filters with speed $\frac{\ell}{\theta}$. Together, they form a linear filter with speed $\frac{\ell}{\theta}$ and relative degree $r$ applied to the output error injection term $\eta$, equivalently written in the compact form

$$
\begin{aligned}
\dot{z} & =-\frac{\ell}{\theta}\left(I_{r}-A_{r}^{\top}\right) z+\frac{\ell}{\theta} C_{r}^{\top}\left(y-C_{n} \hat{x}\right), \\
\eta & =B_{r}^{\top} z,
\end{aligned}
$$

where $r \in \mathbb{N}_{>0}$ is the dimension of the filter (and its relative degree), $z=\left(z_{1}, \ldots, z_{r}\right)^{\top}$ is its state, and matrices $A_{r}, B_{r}$ and $C_{r}$ are defined in (1). It is readily seen that filter (10) is parametrized by the high-gain parameter $\ell$ of the high-gain observer (4) and by a second parameter $\theta>0$ whose design is rather straightforward, as characterized in the next section.

Remark 1 (Different filter positions) The motivation for applying the filtering action directly to $y-\hat{y}$, rather than applying it only to the output $y$ is clearly explained in Figure 3, showing that any filtering action on $y$ should be reproduced also at the output of the high-gain observer, so that the forward invariance of the zero-estimation error manifold is preserved (a necessary condition for asymptotic estimation), as proposed in the case of PI-observers, see, e.g., [9, 10]. This condition can be equivalently interpreted, for linear systems, by noting that the presence of a low-pass filter introduces a high-frequency phase-shift that causes distortions of the estimate, if the filter is not suitably replicated. As a consequence, applying the pink "filter" block to the output $y$ as in Figure 3 requires introducing an additional

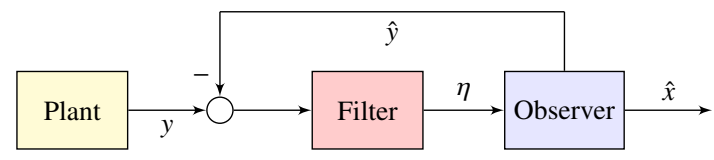

Figure 2: Output injection redesign via linear filtering.

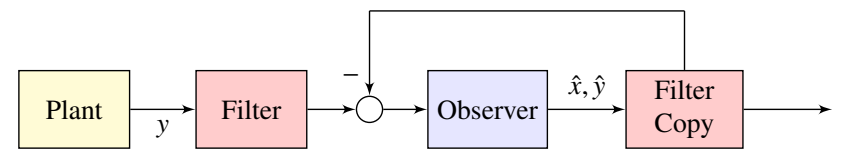

Figure 3: Pre-filtering the measure $y$ before using an observer.

filtering action at the output $\hat{y}$ of the observer (the block called "filter copy" in that figure), resulting in the same scheme as that of Figure 2 via a non-minimal representation, which does not require implementing in the observer powers of $\ell$ of or$\operatorname{der} \ell^{n+r}$. An alternative approach is proposed in [20], where only the measurement $y$ is filtered. However in that case, the asymptotic behavior of the high-gain observer in the absence of measurement noise is only preserved in an output feedback stabilization framework and not in the pure observation context addressed here.

Remark 2 (Overall observer dimension) There are a number of existing results about dynamic augmentations of state observers, such as those proposed in [3], [4], [6], [18], [26], [28]. We highlight next some of the main differences and advantages arising from our approach. First of all, the overall dimension of the augmented observer (4), (10), is $n+r$ where $r \in \mathbb{N}_{>0}$ can be freely selected, to improve the sensitivity to high-frequency measurement noise. Similarly, the dimension of the observer proposed in [26] is $n+r$ but the filter design is more involved than what we propose here. Conversely, in [4] the overall dimension of the observer is fixed as $2 n-2$ and in [3] and [28] the dimension is $2 n$. Finally, in the solution proposed in [18] the overall dimension of the observer is $n$, but the relative degree between the measurement noise and the state estimate is 1 . Instead, our solution (4), (10) shows by design a relative degree of at least $r+1$ (depending on the specific component under consideration), thus resulting in better performance with high-frequency measurement noise, as well characterized later in Section 5.

\section{Stability of the error dynamics}

\subsection{Main stability result}

As mentioned after introducing (10), once the (relative) degree $r \in \mathbb{N}_{>0}$ is fixed, the only parameter that must be selected in our redesign law is the scalar $\theta>0$. We illustrate here a simple tuning rule for this design. The following is our main stability result for the estimation error dynamics. 
Theorem 1 Consider system (3) and a high-gain observer (4) with a matrix gain $K_{n}$ such that $A_{n}-K_{n} C_{n}$ is Hurwitz. Fix the order $r \in \mathbb{N}_{>0}$ of filter (10) and consider the solution of the redesigned observer (4), (10). There exists $\theta^{\star}>0$ such that, for each $\theta \in\left(0, \theta^{\star}\right)$, there exist $\ell^{\star} \geq 1$ and $\alpha, \beta, \gamma>0$ such that, for any $\ell>\ell^{\star}$, bound (7) holds along all solutions starting from $(x(0), \hat{x}(0)) \in \mathcal{X}_{0} \times \mathbb{R}^{n}$ and $z(0)=0$.

We emphasize that the tuning rule characterized by Theorem 1 is intuitive and simpler than the constructions [4], [6], [28] and [26]. We also remark that with $z(0) \neq 0$, bound (7) would simply involve an extra decaying term proportional to $\ell^{i-1}|z(0)|$, due to the transient response of the stable filter.

\subsection{Proof of Theorem 1}

Consider the change of coordinates

$$
(x, \hat{x}, z) \mapsto(x, e, z):=\left(x, \ell D_{n}(\ell)^{-1}(x-\hat{x}), z\right),
$$

with $D_{n}(\ell)$ defined after (5). With $\left(A_{n}, B_{n}, C_{n}\right)$ as in (1), we recall the following properties

$$
\begin{gathered}
D_{n}^{-1}(\ell) A_{n} D_{n}(\ell)=\ell A_{n}, \quad \ell D_{n}^{-1}(\ell) B_{n}=\ell^{-(n-1)} B_{n}, \\
C_{n} \ell^{-1} D_{n}(\ell)=C_{n} .
\end{gathered}
$$

By compactly denoting $\xi:=\left(e^{\top}, z^{\top}\right)^{\top}$, and by using the timerescaling $t \mapsto \tau(t):=\ell t$, similarly to [1], the dynamics of system (4), (10) reads

$$
\frac{d \xi}{d \tau}=F(\theta) \xi+\ell^{-1} H \Delta(e, x, u)+\theta^{-1} G v
$$

where we defined

$$
\begin{aligned}
& F(\theta):=\left(\begin{array}{cc}
A_{n} & -K_{n} B_{r}^{\top} \\
\frac{1}{\theta} C_{r}^{\top} C_{n} & -\frac{1}{\theta}\left(I_{r}-A_{r}^{\top}\right)
\end{array}\right), \begin{array}{l}
G:=\left(0_{1 \times n}, C_{r}\right)^{\top}, \\
H:=\left(B_{n}^{\top}, 0_{1 \times r}\right)^{\top},
\end{array} \\
& \Delta(e, x, u):=\frac{1}{\ell^{n-1}}\left[\varphi(x, u)-\varphi_{s}\left(x-\ell^{-1} D_{n}(\ell) e, u\right)\right] .
\end{aligned}
$$

In view of (6), if $\ell \geq 1$, we have $|\Delta(e, x, u)| \leq \bar{\varphi}|e|$, for all $x \in \mathcal{X}$, $e \in \mathbb{R}^{n}$ and $u \in \mathcal{U}$. The coordinate system (13) and specifically the structure of matrix $F(\theta)$ in (14) are key to proving Theorem 1 , specifically through the following result, whose proof is postponed to the end of the section, to avoid breaking the flow of the exposition.

Lemma 1 Let $K_{n}$ be such that $A_{n}-K_{n} C_{n}$ is Hurwitz. Then, for each $r \in \mathbb{N}_{>0}$, there exists $\theta^{\star}>0$ such that, for any $\theta \in\left(0, \theta^{\star}\right)$, the matrix $F(\theta)$ in (14) is Hurwitz. Moreover, there exists a symmetric positive definite matrix $P(\theta) \in \mathbb{R}^{(n+r) \times(n+r)}$ such that

$$
P(\theta) F(\theta)+F^{\top}(\theta) P(\theta)=-I_{n+r} .
$$

The matrix $P(\theta)$ is structured as follows:

$$
P(\theta)=\left(\begin{array}{cc}
P_{0}+r \tilde{P}_{0} & 0_{n, r} \\
0_{r, n} & 0_{r}
\end{array}\right)+O(\theta),
$$

where $O(\theta)$ is a symmetric matrix whose entries are small terms of order $\theta$, and $P_{0} \in \mathbb{R}^{n \times n}$ and $\tilde{P}_{0} \in \mathbb{R}^{n \times n}$ are respectively the unique symmetric positive definite solutions to

$$
\begin{aligned}
& P_{0}\left(A_{n}-K_{n} C_{n}\right)+\left(A_{n}-K_{n} C_{n}\right)^{\top} P_{0}=-I_{n}, \\
& \tilde{P}_{0}\left(A_{n}-K_{n} C_{n}\right)+\left(A_{n}-K_{n} C_{n}\right)^{\top} \tilde{P}_{0}=-C_{n}^{\top} C_{n} .
\end{aligned}
$$

In order to complete the proof of Theorem 1 , denote by $p$ and $\bar{p}$ the smallest and the largest eigenvalues of $P(\theta)$ defined in Lemma 1 and consider the Lyapunov function $V(\xi)=\xi^{\top} P(\theta) \xi$. Differentiating $V$ along the solutions of (13) yields

$$
\frac{d}{d \tau} V(\xi)=2 \xi^{\top} P(\theta)\left(F(\theta) \xi+\ell^{-1} H \Delta(\xi, x, u)+\theta^{-1} G v\right) .
$$

In order to upper bound the latter derivative, consider separately the three terms (see, e.g., [1]). Thanks to (16), we have $2 \xi^{\top} P(\theta) F(\theta) \xi=-|\xi|^{2}$. By using the inequality $|e| \leq|\xi|$ and the Lipschitz properties of $\Delta$, we have

$$
2 \ell^{-1}\left|\xi^{\top} P(\theta)\right||H \Delta| \leq 2 \ell^{-1} \bar{p}|\xi| \bar{\varphi}|\xi|
$$

Finally, the following Young inequality

$2\left|\xi^{\top} P(\theta) \theta^{-1} G v\right| \leq\left|\xi^{\top}\right|\left|2 \theta^{-1} P(\theta) G v\right|$,

$$
\leq \frac{1}{2}|\xi|^{2}+\theta^{-2} \frac{1}{2}|2 P(\theta) G v|^{2}, \leq \frac{1}{2}|\xi|^{2}+\frac{2 \bar{p}^{2}}{\theta^{2}}|v|^{2}
$$

leads to

$$
\frac{d}{d \tau} V(\xi) \leq-\frac{1}{2}\left(1-4 \ell^{-1} \bar{p} \bar{\varphi}\right)|\xi|^{2}+c_{1}|v|^{2}
$$

where $c_{1}=2 \bar{p}^{2} / \theta^{2}$. Now, choose any $\ell^{\star}>\max \{1,4 \bar{\varphi} \bar{p}\}$ so that there exists $\beta>0$ satisfying, for any $\ell \geq \ell^{\star}$, the inequality $1-4 \ell^{-1} \bar{\varphi} \bar{p} \geq 4 \beta \bar{p}$. Then we obtain, for all $\ell \geq \ell^{\star}$,

$$
\frac{d}{d \tau} V(\xi(\tau)) \leq-2 \beta V(\xi(\tau))+c_{1}|v(\tau)|^{2}
$$

Hence, by using the comparison principle, we have

$$
\begin{aligned}
V(\xi(\tau)) & \leq \exp (-2 \beta \tau) V(\xi(0))+\int_{0}^{\tau} \exp (-2 \beta(\tau-s)) c_{1}|v(s)|^{2} \mathrm{~d} s \\
& \leq \exp (-2 \beta \tau) V(\xi(0))+c_{2}\|v\|_{\infty}^{2} .
\end{aligned}
$$

where $c_{2}=c_{1} /(2 \beta)=\bar{p}^{2} /\left(\theta^{2} \beta\right)$. Recall now that $z(0)=0$ and use the following inequalities, derived from (11), and $\ell \geq 1$,

$$
\left|\hat{x}_{i}-x_{i}\right| \leq \ell^{i-1}\left|e_{i}\right| \leq \ell^{i-1}|\xi|, \quad|\xi|^{2} \leq|\hat{x}-x|^{2}+|z|^{2} .
$$

By using (20), the definition of $V$, and $\tau=\ell t$, we therefore obtain (7), with $\alpha=\sqrt{\bar{p} / \underline{p}}, \gamma=\sqrt{c_{2} / \underline{p}}=\bar{p} /(\theta \sqrt{\underline{p} \beta})$. Note that the values of $\ell^{\star}, \alpha$ and $\gamma$ do not depend on $\ell$ but depend, in general, on $\theta$ because of $F(\theta)$ in (14), and therefore of $P(\theta)$.

\subsection{Proof of Lemma 1}

Let $r \in \mathbb{N}_{>0}$ be fixed and consider the matrix $F(\theta)$ defined by (14). We recognize in $F(\theta)$ a structure found in singularly perturbed linear systems and follow the approach in [21] to investigate its stability for a small enough parameter $\theta$. Before establishing the result, let us investigate the Parametrized Nonsymmetric Algebraic Riccati Equation (PNARE), in the parameter $\theta$ and the rectangular variable $L(\theta) \in \mathbb{R}^{r \times n}[17,16]$ :

$$
C_{r}^{\top} C_{n}-\left(I_{r}-A_{r}^{\top}\right) L(\theta)-\theta L(\theta) A_{n}+\theta L(\theta) K_{n} B_{r}^{\top} L(\theta)=0_{r, n} .
$$


For $\theta=0$, the PNARE (21) becomes affine in $L$ and admits a unique solution $L(0)=\left(I_{r}-A_{r}^{\top}\right)^{-1} C_{r}^{\top} C_{n}=\mathbb{1}_{r} C_{n}$, because $\left(I_{r}-A_{r}^{\top}\right)$ is invertible (it is lower triangular).

Following [17], we may denote the left-hand side of (21) as $f(M(\theta), L(\theta))$, where the characteristic matrix $M(\theta)$ is given by

$$
\begin{aligned}
& \left(\begin{array}{c:c}
M_{11}(\theta) & M_{12}(\theta) \\
\hdashline M_{21}(\theta) & M_{22}(\theta)
\end{array}\right)=\theta F(\theta) \\
& \quad=\left(\begin{array}{c:c}
\theta A_{n} & -\theta K_{n} B_{r}^{\top} \\
\hdashline C_{r}^{\top} C_{n} & -\left(\bar{I}_{r}-\bar{A}_{r}^{\top}\right)
\end{array}\right) \in \mathbb{R}^{(n+r) \times(n+r)},
\end{aligned}
$$

which satisfies

$$
\left.\left(\begin{array}{ll}
-L(\theta) & I_{r}
\end{array}\right) M(\theta)\left(\begin{array}{c}
I_{n} \\
L(\theta)
\end{array}\right)=f(M(\theta), L(\theta))\right)
$$

Observe now that $\sigma\left(M_{11}(0)+M_{12}(0) L(0)\right) \quad \cap$ $\sigma\left(M_{22}(0)-L(0) M_{12}(0)\right)=\sigma\left(0_{n, n}\right) \cap \sigma\left(A_{r}^{\top}-I_{r}^{\top}\right)=\emptyset$, therefore the assumptions of [17, Lemma 2.3] are satisfied and there exist an open neighborhood $\Theta$ of 0 and a neighborhood ${ }^{2}$ $\mathcal{N} \subset \mathbb{R}^{r \times n}$ of $L(0)$ such that for each $\theta \in \Theta$, the PNARE (21) has a unique solution $L(\theta) \in \mathcal{N}$. Moreover, $L(\theta)$ is differentiable with respect to $\theta$ and admits the zero-order Taylor series: $L(\theta)=\mathbb{1}_{r} C_{n}+O(\theta)$.

Introduce now the Parametrized Non-symmetric Algebraic Sylvester Equation (a special case of the PNARE), in the parameter $\theta$ and the rectangular variable $X(\theta) \in \mathbb{R}^{n \times r}$ :

$$
\begin{aligned}
& \theta\left(A_{n}-K_{n} B_{r}^{\top} L(\theta)\right) X(\theta)-\theta K_{n} B_{r}^{\top} \\
& \quad+X(\theta)\left(\left(I_{r}-A_{r}\right)^{\top}-\theta L(\theta) K_{n} B_{r}^{\top}\right)=0_{n, r} .
\end{aligned}
$$

Thanks to the same arguments as for the PNARE (21), we conclude that, for $\theta=0$, Equation (24) becomes affine in $X$ and admits a unique solution $X(0)=0_{n, r}$. In addition, there exist an open neighborhood $\Theta$ of $\theta=0$ (which we may consider to be the same as the one above, without loss of generality) and a neighborhood of $X(0)=0_{n, r}$ such that, for each $\theta \in \Theta$, Equation (24) admits a unique and differentiable solution $X(\theta)=\theta \tilde{X}(\theta)$, for a suitable matrix function $\tilde{X}$.

Let us use these solutions $L(\theta)$ and $\theta \tilde{X}(\theta)$ to apply the Chang transformation

$$
\begin{aligned}
T(\theta) & =\left(\begin{array}{cc}
I_{n} & -X(\theta) \\
0_{r, n} & I_{r}
\end{array}\right)\left(\begin{array}{cc}
I_{n} & 0_{n, r} \\
-L(\theta) & I_{r}
\end{array}\right), \\
& =\left(\begin{array}{cc}
I_{n}-\theta \tilde{X}(\theta) L(\theta) & -\theta \tilde{X}(\theta) \\
L(\theta) & I_{r}
\end{array}\right),
\end{aligned}
$$

introduced in [11] and which is invertible:

$$
\begin{aligned}
T^{-1}(\theta) & =\left(\begin{array}{cc}
I_{n} & 0_{n, r} \\
L(\theta) & I_{r}
\end{array}\right)\left(\begin{array}{cc}
I_{n} & X(\theta) \\
0_{r, n} & I_{r}
\end{array}\right) \\
& =\left(\begin{array}{cc}
I_{n} & -\theta \tilde{X}(\theta) \\
L(\theta) & I_{r}+\theta \tilde{X}(\theta) L(\theta)
\end{array}\right)
\end{aligned}
$$

\footnotetext{
${ }^{2}$ The neighborhoods are complex in [17] but can be projected on $\mathbb{R}^{r \times n}$.
}

We obtain, by exploiting (21) and (24),

$$
\begin{aligned}
\tilde{F}(\theta) & =T(\theta) F(\theta) T^{-1}(\theta), \\
& =\left(\begin{array}{cc}
A_{n}-K_{n} B_{r}^{\top} L(\theta) & 0_{n, r} \\
0_{r, n} & -\theta^{-1}\left(I_{r}-A_{r}^{\top}\right)+L(\theta) K_{n} B_{r}^{\top}
\end{array}\right), \\
& =\left(\begin{array}{cc}
A_{n}-K_{n} C_{n}+O(\theta) & 0_{n, r} \\
0_{r, n} & -\theta^{-1}\left(I_{r}-A_{r}^{\top}\right)+O(1)
\end{array}\right) .
\end{aligned}
$$

Since $A_{n}-K_{n} C_{n}$ and $-\left(I_{r}-A_{r}^{\top}\right)$ are Hurwitz by construction, the matrix $F(\theta)$ in (14) is Hurwitz for $\theta$ small enough.

Let us now focus on the structure of the matrix $P$. The Lyapunov equation (16) is equivalent to

$$
\tilde{P}(\theta) \tilde{F}(\theta)+\tilde{F}^{\top}(\theta) \tilde{P}(\theta)=-T^{-\top}(\theta) T^{-1}(\theta),
$$

with $\tilde{P}(\theta)=T^{-\top}(\theta) P(\theta) T^{-1}(\theta)$. By noticing that, from (26),

$$
T^{-\top}(\theta) T^{-1}(\theta)=\left(\begin{array}{cc}
I_{n}+L^{\top}(0) L(0) & L^{\top}(0) \\
L(0) & I_{r}
\end{array}\right)+O(\theta)
$$

and by considering the block diagonal structure of $\tilde{F}(\theta)$ in (27), we can follow [21, Section 2.6] (see, in particular, [21, eq. (6.36)]) to prove that the next structured matrix

$$
\tilde{P}(\theta)=\left(\begin{array}{cc}
\tilde{P}_{1}(\theta) & \theta \tilde{P}_{2}(\theta) \\
\theta \tilde{P}_{2}^{\top}(\theta) & \theta \tilde{P}_{3}(\theta)
\end{array}\right)
$$

satisfies the Lyapunov equation (28), where $\tilde{P}_{1}(\theta)$ is the solution of the Lyapunov equation

$$
\begin{aligned}
& \tilde{P}_{1}(\theta)\left(A_{n}-K_{n} B_{r}^{\top} L(\theta)\right)+\left(A_{n}-K_{n} B_{r}^{\top} L(\theta)\right)^{\top} \tilde{P}_{1}(\theta)= \\
& \quad=-\left(I_{n}+L^{\top}(0) L(0)\right)+O(\theta)=-\left(I_{n}+r C_{n}^{\top} C_{n}\right)+O(\theta)
\end{aligned}
$$

Returning to the original basis, $P(\theta)=T^{\top}(\theta) \tilde{P}(\theta) T(\theta)$ and using the linearity of the Lyapunov equation, the proof is completed.

\section{Performance with high-frequency noise}

For the high-gain observer (4)-(5), it is shown in [5] that the steady-state behavior of the estimation error in the presence of high-frequency measurement noise (8) can be characterized as a low-pass filter behavior of order 1, as in (9). Furthermore, the proof technique proposed in [5] has been applied to low-power high-gain observers in [4] and [6], to show how to capture the relative degree effect in the characterization of the steady-state behavior (9). As a consequence, by following the ideas proposed in [5], [6], we show below that the high-gain observer (4) with filter (10) improves the bound (9) in the sense characterized next.

Theorem 2 Consider system (3) and observer (4), (10), and any stabilizing selection of $K_{n}, r, \theta$ and $\ell$, according to Theorem 1. Then there exists $\varepsilon^{\star}>0$ such that, for any $\varepsilon \in\left(0, \varepsilon^{\star}\right)$, the closed loop perturbed by the measurement noise $v$ in (8), with the frequencies $\omega_{i}$ being pairwise incommensurable, the following bound holds

$$
\limsup _{t \rightarrow+\infty}\left|x_{i}(t)-\hat{x}_{i}(t)\right| \leq\left(\frac{\varepsilon \ell}{\theta}\right)^{r} \varepsilon \varrho \ell^{i}\|v\|_{\infty}, \forall i=1 \ldots, n,
$$


for all initial conditions $(x(0), \hat{x}(0), z(0)) \in \mathcal{X}_{0} \times \mathbb{R}^{n} \times \mathbb{R}^{r}$, and for some $\varrho>0$ independent of $\theta, \ell$.

Theorem 2 establishes that, once parameters $\ell$ and $\theta$ have been selected according to Theorem 1 to ensure convergence to zero of the estimation error, the asymptotic norm of the estimation error at high frequencies (namely as $\varepsilon \rightarrow 0$ ) goes to zero increasingly fast for larger values of $r$ (due to the term $\left.\varepsilon^{r+1}\right)$. Similar conclusions were drawn in [6]. Comparing the bound (9) given by the standard high-gain observer (4), (5), with the bound (29) one may also see that the proposed filtering action negatively affects the estimate due to the term $\left(\frac{\ell}{\theta}\right)^{r}$ (note that $\ell$ is typically large and $\theta$ is typically small, as characterized in Theorem 1). However, due to the effect of the relative degree, the asymptotic gain is also multiplied by $\varepsilon^{r+1}$ (while in (9) there is only $\varepsilon$ ), thus mitigating the total impact of the noise $v$ on the estimation error at high-frequencies (i.e. for small $\varepsilon$ ). Finally, note that, for $r=0$, namely when no filter is used, the inequality (29) boils down to (9), thus recovering the asymptotic properties of the standard high-gain observer (4)-(5).

As in [5], to prove Theorem 2, we represent the measurement noise (8) as the output of the following autonomous system

$$
\varepsilon \dot{w}=S w, \quad v=R w,
$$

where $S$ is a neutrally stable matrix (having simple eigenvalues on the imaginary axis) and $R$ is a row vector. System (30) can be conveniently seen as generator of $N>0$ harmonics at frequencies $\omega_{i} / \varepsilon>0, i=1, \ldots, N$, namely, the matrix $S$ is a block diagonal matrix of the form

$$
S:=\operatorname{blkdiag}\left(S_{1}, \ldots, S_{N}\right), \quad S_{i}:=\left(\begin{array}{cc}
0 & \omega_{i} \\
-\omega_{i} & 0
\end{array}\right)
$$

and $R:=\left(\left(\begin{array}{ll}0 & 1\end{array}\right)\left(\begin{array}{llll}0 & 1\end{array}\right) \cdots\left(\begin{array}{ll}0 & 1\end{array}\right)\right)$. In the following we assume that $w=\operatorname{col}\left(w_{1}, \ldots, w_{N}\right)$ ranges in a compact invariant set $\mathcal{W} \subset$ $\mathbb{R}^{2 N}$. The assumption stated in Theorem 2 that frequencies $\omega_{i}$ are pairwise incommensurable, is motivated by the fact that we may conveniently represent the overall size of the oscillators as the $\mathcal{L}_{\infty}$ norm of the output $v$, namely

$$
\|v\|_{\infty}=\sum_{i=1}^{N} v_{i}=\sum_{i=1}^{N}\left|w_{i}(t)\right|=\sum_{i=1}^{N}\left|w_{i}(0)\right|, \quad \forall t \geq 0 .
$$

The first equality in (31) (existence of the supremum and its value) comes from a property of quasi-sinusoidal signals (see for instance [25, Lemma 1, Theorem 1 and eq. (4.3) page 11]), which is the case of the noise $v$, defined in (8) with pairwise incommensurable frequencies $\omega_{i}$. The other equalities in (31) are obtained by recalling that, being $w_{i} \in \mathbb{R}^{2}$ the state of each oscillator, then $t \mapsto\left|w_{i}(t)\right|=v_{i}$ is constant for each $i=1, \ldots, N$. Equality (31) helps in dealing with the right hand side of (29) based on the properties of the exosystem (30). In particular, in the proof of Theorem 2 we use the following "observabilitylike" property

$$
|w(0)|=|w(t)| \leq \sum_{i=1}^{N}\left|w_{i}(t)\right|=\|v\|_{\infty}, \quad \forall t \geq 0,
$$

obtained from standard properties of vector norms. Again, we emphasize that the Euclidean norm $t \mapsto|w(t)|$ is constant along the revolving solutions of (30).

Proof of Theorem 2. The proof follows the same argument presented in [5], adapted to the structure (4), (10). As shown in [5], it is enough to analyze the "linear" case $\varphi(x, u)=\varphi_{s}(x, u)=\Phi x$, with $|\Phi| \leq \bar{\varphi}$. Indeed, it is shown in [5] that the obtained bounds can be extended also to the nonlinear context. The corresponding derivations are omitted because they would re-propose the same arguments as those developed in [5] for the high-gain architecture (4)-(5) and in [6] for the low-power high-gain architecture.

Within this linear setting, consider the change of coordinates $(x, \hat{x}) \mapsto(x, \tilde{x}):=(x, x-\hat{x})$. Then observer $(4),(10)$, forced by system (30) reads

$$
\begin{aligned}
& \dot{\tilde{x}}=\left(A_{n}+B_{n} \Phi\right) \tilde{x}+D_{n}(\ell) K_{n} B_{r}^{\top} z, \\
& \dot{z}=-\frac{\ell}{\theta}\left(I_{r}-A_{r}^{\top}\right) z+\frac{\ell}{\theta} C_{r}^{\top} C_{n} \tilde{x}+\frac{\ell}{\theta} C_{r}^{\top} R w,
\end{aligned}
$$

which can be written in compact from, using $\tilde{\xi}:=\operatorname{col}(\tilde{x}, z)$, as

$$
\begin{aligned}
\varepsilon \dot{w} & =S w, \\
\dot{\tilde{\xi}} & =\Lambda \tilde{\xi}+\Gamma R w, \\
& \text { where } \Lambda:=\left(\begin{array}{cc}
A_{n}+B_{n} \Phi & -D_{n}(\ell) K_{n} B_{r}^{\top} \\
\frac{\ell}{\theta} C_{r}^{\top} C_{n} & -\frac{\ell}{\theta}\left(I_{r}-A_{r}^{\top}\right)
\end{array}\right), \quad \Gamma:=\frac{\ell}{\theta}\left(\begin{array}{c}
0_{n \times 1} \\
C_{r}^{\top}
\end{array}\right) .
\end{aligned}
$$

Let us now show that matrix $\Lambda$ is Hurwitz by way of equivalently showing that (we use below the identities in (12)) the transformed matrix

$$
\begin{aligned}
\bar{\Lambda} & :=\ell^{-1} \operatorname{diag}\left(\ell D_{n}^{-1}(\ell), I_{r}\right) \Lambda \operatorname{diag}\left(\ell^{-1} D_{n}(\ell), I_{r}\right) \\
& =\left(\begin{array}{cc}
A_{n}+B_{n} \ell^{-1} \Phi D_{n}(\ell) \ell^{-n} & -K_{n} B_{r}^{\top} \\
\frac{1}{\theta} C_{r}^{\top} C_{n} & -\frac{1}{\theta}\left(I_{r}-A_{r}^{\top}\right)
\end{array}\right)
\end{aligned}
$$

is Hurwitz for small enough $\theta$. To this end, since parameters $K_{n}, r, \theta, \ell$ are selected according to Theorem 1 , using the notation in the proof of Theorem 1 and denoting $\xi:=\operatorname{col}(e, z)$, we may write $\bar{\Lambda} \xi=F \xi+\ell^{-1} H \delta$, where $\delta:=\Phi D_{n}(\ell) \ell^{-n} e$ satisfies $|\delta| \leq \bar{\varphi}|e|$, due to the assumptions on $\Phi$. We may then follow the same steps as those leading to (20) to show that $2 \xi^{\top} P \bar{\Lambda} \xi<0$ for all $\xi \neq 0$, with $P>0$ given by (16). By standard linear stability theory, this proves that $\bar{\Lambda}$ (therefore $\Lambda$ ) is Hurwitz.

Since $\Lambda$ is Hurwitz, from standard regulation theory [15] the steady-state response of system (33) is given by $\tilde{\xi}_{\mathrm{ss}}:=\Pi_{\mathcal{E}} w$, where $\Pi_{\varepsilon}$ solves the following Sylvester equation (the regulator equation)

$$
\Pi_{\mathcal{E}} S=\varepsilon \Lambda \Pi_{\varepsilon}+\varepsilon \Gamma P .
$$

Indeed, if there exists such a $\Pi_{\varepsilon}$, the dynamics of $\left(\tilde{\xi}-\Pi_{\mathcal{\varepsilon}} w\right)$ is given by

$$
\frac{d}{d t}\left(\tilde{\xi}-\Pi_{\varepsilon} w\right)=\Lambda\left(\tilde{\xi}-\Pi_{\varepsilon} w\right),
$$

and stability of $\Lambda$ implies asymptotically convergence of $\tilde{\xi}-$ $\Pi_{\varepsilon} w$ to zero. Now, the Sylvester equation (34) admits a unique solution because the eigenvalues of $S$ are all on the imaginary axis while the ones of $\Lambda$ are in the negative real half plane (they 
are therefore disjoint). As discussed in [5], since $S$ is not singular, the unique solution of (34) is given by

$$
\Pi_{\varepsilon}=\sum_{k=1}^{+\infty} \varepsilon^{k} \bar{\Pi}_{k}, \quad \bar{\Pi}_{k}=\Lambda^{k-1} \Gamma R S^{-k} .
$$

In (35), the series is convergent as long as $\varepsilon$ is taken sufficiently small, because $\left|\Pi_{\varepsilon}\right| \leq\left|\Lambda^{-1} \Gamma R\right| \sum_{k=1}^{+\infty}(\varepsilon|\Lambda||S|)^{k}$, leading to a geometric series if $\varepsilon^{-1}$ is larger than the product of the spectral radii of $\Lambda$ and $S$. Moreover, to prove that (35) is a solution (therefore the unique solution) of (34) it suffices to plug it into (34) to get

$$
\begin{aligned}
\Pi_{\varepsilon} S & =\sum_{k=1}^{+\infty} \varepsilon^{k} \Lambda^{k-1} \Gamma R S^{-k+1}=\varepsilon \Gamma R+\sum_{k=2}^{+\infty} \varepsilon^{k} \Lambda^{k-1} \Gamma R S^{-k+1} \\
& =\varepsilon \Gamma R+\sum_{h=1}^{+\infty} \varepsilon^{h+1} \Lambda \Lambda^{h-1} \Gamma R S^{-h}=\varepsilon \Gamma R+\varepsilon \Lambda \Pi_{\varepsilon}
\end{aligned}
$$

Let us now focus on (29) and observe that, with $\mathfrak{e}_{i}$ denoting the $i$-th column of the identity matrix, we obtain $\mathfrak{e}_{i}^{\top} \tilde{\xi}=\tilde{x}_{i}=x_{i}-\hat{x}_{i}$, $i=1, \ldots, n$. In particular, since $\lim _{t \rightarrow+\infty}\left(\tilde{\xi}(t)-\Pi_{\mathcal{\varepsilon}} w(t)\right)=0$ (by the definition of steady-state behavior), we have that

$$
\limsup _{t \rightarrow+\infty}\left|x_{i}(t)-\hat{x}_{i}(t)\right|=\limsup _{t \rightarrow+\infty}\left|\mathfrak{e}_{i}^{\top} \Pi_{\varepsilon} w(t)\right|, i=1, \ldots, n .
$$

To establish a bound on the right-hand side of (36), we use the expression given by (35), obtaining

$$
\left|\mathfrak{e}_{i}^{\top} \Pi_{\varepsilon}\right|=\left|\mathfrak{e}_{i}^{\top} \sum_{k=1}^{+\infty} \varepsilon^{k} \Lambda^{k-1} \Gamma R S^{-k}\right| .
$$

We need to analyze more carefully the structure of the righthand side of (37). From (1), we easily get $C_{r}\left(I_{r}-A_{r}^{\top}\right)^{k-1} B_{r}=0$ for all $k=1, \ldots, r-1$ and $C_{r}\left(I_{r}-A_{r}^{\top}\right)^{r-1} B_{r}=1$. Therefore, using the expressions in (33) we get

$$
\begin{aligned}
& \mathfrak{e}_{i}^{\top} \Lambda^{k-1} \Gamma=\mathfrak{e}_{i}^{\top}\left(\begin{array}{c}
0_{n \times 1} \\
\left(\frac{\ell}{\theta}\right)^{k}\left(I_{r}-A_{r}^{\top}\right)^{k-1} C_{r}^{\top}
\end{array}\right)=0, \quad \forall k=1, \ldots, r \\
& \mathfrak{e}_{i}^{\top} \Lambda^{r} \Gamma=\mathfrak{e}_{i}^{\top}\left(\begin{array}{c}
D_{n}(\ell) K_{n}\left(\frac{\ell}{\theta}\right)^{r} \\
\left(\frac{\ell}{\theta}\right)^{r+1}\left(I_{r}-A_{r}^{\top}\right)^{r} C_{r}^{\top}
\end{array}\right)=\ell^{i} k_{i}\left(\frac{\ell}{\theta}\right)^{r},
\end{aligned}
$$

for all $i=1, \ldots, n$. Hence, combining bounds (37) with expression (38) gives, for all $i=1, \ldots, n$,

$$
\left|\mathfrak{e}_{i}^{\top} \Pi_{\varepsilon}\right|=\varepsilon^{r+1} \ell^{i} k_{i}\left(\frac{\ell}{\theta}\right)^{r}\left|R S^{-(r+1)}\right|+\sum_{k=r+2}^{+\infty} \varepsilon^{k}\left|\mathfrak{e}_{i}^{\top} \bar{\Pi}_{k}\right|
$$

Since matrices $\bar{\Pi}_{k}$ in (35) do not depend on $\varepsilon$, for $\varepsilon$ small enough, the dominant terms in (39) are the ones with smallest power of $\varepsilon$. As a consequence, by combining (39) with (36), and also using bound (32), we directly obtain bound (29) for $\varepsilon$ sufficiently small, for a scalar $\varrho$ independent of $\ell, \theta, \varepsilon$.

\section{Numerical Discussion}

For a nominal high-gain observer (4), (5), it can be proved (see, for instance, [19]) that the minimum value of $\ell$ to ensure bound (7) is proportional to the maximum eigenvalue $\bar{p}_{0}=\lambda_{\max }\left(P_{0}\right)$ of the symmetric positive definite matrix $P_{0}$ solution to (18). For the proposed observer (4), (10), the minimum value of $\ell^{\star}$ is proportional to the maximum eigenvalue $\bar{p}=\bar{p}(\theta)=\lambda_{\text {max }}(P(\theta))$ of the matrix $P(\theta)$ solution to (16). Such a value depends in general on the choice of $\theta$. Furthermore, as shown in Lemma 1, this choice is critical as large values of $\theta$ may lead to instability of $F$.

As a consequence, we investigate numerically the following aspects: 1) the impact of the dimension of the filter $r$ on the choice of $\theta$;2) the impact of $\theta$ on $\bar{p}$; 3) the limit of $\bar{p}$ as $\theta$ converges to zero; 4) a possible optimal selection for $\theta$.

We recall that the literature dealing with upper and lower bounds of the maximum eigenvalue of the positive definite solution of a Lyapunov equation (16) is rich and may be conservative, as emphasized in the survey [22]. As a consequence, to facilitate the qualitative discussion about these questions, we study here the impact of $r$ and $\theta$ on $\bar{p}$ via numerical simulations.

As a simple example, we consider $n=3$, and $K_{3}=(6,11,6)^{\top}$ in (4) so that the eigenvalues of $\left(A_{3}-K_{3} C_{3}\right)$ are $\{-1,-2,-3\}$. We compute the $\theta^{\star}$ of Lemma 1 as the largest real number ensuring that $F$ in (14) is still Hurwitz. The values for $r=1,2,3$ are reported in Table 1. The values of $\bar{p}$ as a function of $\theta$, for $r=1,2,3$, are depicted in Figure 4. Such graphs confirm that when $\theta \rightarrow \theta^{\star}$ then $\bar{p}(\theta)$ tends to $+\infty$ (as expected since $F(\theta)$ tends to instability). In addition, thanks to the structure of $P(\theta)$ given in (17), we verify that, when $\theta \rightarrow 0, \bar{p}(\theta)$ converges to $\lambda_{\max }\left(P_{0}+r \tilde{P}_{0}\right)>\bar{p}_{0}$, due to the positive definiteness of $\tilde{P}_{0}$. Numerical simulations for different selections of $n, r$ and eigenvalues of the matrix $A_{n}-K_{n} C_{n}$ (for instance with some imaginary part) confirmed the same type of results and curves.

Then, in order to find a criterion to select an optimal $\theta$, we focus on the ratio characterizing the high-frequency asymptotic gain in (29). Since $\ell$ is proportional to $\bar{p}$, it is reasonable to look at the ratio

$$
\frac{\bar{p}^{n+r}(\theta)}{\theta^{r}} .
$$

Then, a possible optimal choice $\theta_{\text {opt }}$ is the minimizer of (40). The graph of (40) for $r=1,2,3$, is depicted in Figure 5. For the considered example, the numerical values of $\theta_{o p t}$ and the corresponding value of $\bar{p}\left(\theta_{\text {opt }}\right)$ minimizing (40) are reported in Table 1. Similar curves have been obtained also for different choices of $n, r$ and $K_{n}$.

Finally, in order to compare the proposed technique (4), (10) to the standard high-gain observer (4), (5), we consider the Rössler (chaotic) oscillator [12, §3.3] described by

$$
\begin{aligned}
\dot{x}_{1} & =a x_{1}+x_{2} \\
\dot{x}_{2} & =-x_{1}+x_{3} \\
\dot{x}_{3} & =u-b x_{3}+x_{2} x_{3} \\
y & =x_{1}+v
\end{aligned}
$$

which is chaotic for $a=0.5, b=3$ and $u(t)=-1, \forall t \geq 0$. The initial conditions of (41) are selected as $(2,-3,4)^{\top}$ and the 


\begin{tabular}{ccccc}
\hline & HGO & $r=1$ & $r=2$ & $r=3$ \\
\hline$\theta^{\star}$ & - & 0.495 & 0.149 & 0.087 \\
\hline$\theta_{\text {opt }}$ & - & 0.130 & 0.061 & 0.039 \\
\hline $\bar{p}\left(\theta_{\text {opt }}\right)$ & - & 2.876 & 3.133 & 3.368 \\
\hline$\lambda_{\max }\left(P_{0}+r \tilde{P}_{0}\right)$ & - & 2.3888 & 2.4571 & 2.5276 \\
\hline $\bar{p}_{0}$ & 2.32 & - & - & - \\
\hline $\limsup _{t \rightarrow+\infty}|x(t)-\hat{x}(t)|$ & 4.85 & 0.34 & 0.11 & 0.067 \\
\hline
\end{tabular}

Table 1: Comparison between a high-gain observer (HGO) (4), (5) with a filtered one (4), (10), for $r=1,2,3$. The table provides the largest $\theta^{\star}$ ensuring stability of $F(\theta)$ in (14), and the values of the optimal section of $\theta_{\text {opt }}$ minimizing the ratio (40) and the corresponding value of the largest eigenvalue $\bar{p}\left(\theta_{\text {opt }}\right)$ of the matrix $P\left(\theta_{\text {opt }}\right)$ defined in (16). The largest eigenvalue $\lambda_{\max }\left(P_{0}+r \tilde{P}_{0}\right)$ is provided to check the limit of $\bar{p}(\theta)$ when $\theta$ tends to 0 . For the HGO, the value $\bar{p}_{0}=\lambda_{\max }\left(P_{0}\right)$ is computed with the Lyapunov equation (18). Finally, the last row provides the asymptotic error amplitude of the different observers estimating system (41) with measurement noise generated as (8), (42).

measurement noise is generated as in (8) with

$$
\begin{array}{llll}
N=2 . & v_{1}=3, & \omega_{1}=1, & \phi_{1}=0, \\
\varepsilon=0.004, & v_{2}=5, & \omega_{2}=\sqrt{3}, & \phi_{2}=0 .
\end{array}
$$

We compare the behavior of a standard high-gain observer (4), (5) with the filter redesign (10) for $r=1,2,3$. As before, we select $K_{3}=(6,11,6)^{\top}$, while $\theta$ is selected as in Table 1 . Such values have been taken in order to minimize the ratio (40), as shown in Figure 5. The high-gain parameter is selected in all the scenarios as $\ell=3$. Since convergence of both observers is guaranteed for any initial condition, without loss of generality, we select $\hat{x}(0)=0$ and $z(0)=0$. Figure 6 depicts the transient behavior of the norm of the estimation error $|x(t)-\hat{x}(t)|$ of all the observers. Note that the peaking phenomenon ${ }^{3}$ is augmented: indeed, the value of $\alpha$ in (7) is proportional to $\bar{p}$, which is larger in the presence of the filters, as reported in Table 1. Then, Figures 7,8, and 9, depict the behaviors of the high-gain observer (4), (5) and the proposed redesign (4), (10) for $r=1,2$, showing the advantages in using the low-pass filter. The asymptotic values for all the considered cases are also reported in Table 1.

Comparisons with alternative schemes using filtered versions of high-gain observers are not reported in this work, as it would be difficult to perform a fair parameter selection (positions of the poles, choice of the high-gain parameter, and so on). Furthermore, this is out of scope as our objective, discussed in the introduction, is to propose a simple "plug-and-play" design without any claim of outperforming existing techniques.

From the numerical results we may draw some preliminary conclusions on the general impact of different choices of $r$. First of all, Figures 4 and 5 clearly illustrate the fact that augmenting $r$ leads to higher values of $\ell^{\star}$. On the other hand, augmenting $r$ desirably reduces the asymptotic gain with respect to highfrequency measurement noise, as predicted by the term $\varepsilon^{r}$ in

\footnotetext{
${ }^{3}$ The peaking phenomenon is a large deviation of the estimation error caused by the wrong choice of initial conditions and is typical of standard high-gain observers, see [19] after equation (8). Such a behavior can be observed in Figure 6 during the first 0.5 seconds of simulations.
}

in equation (29). As a consequence, a trade-off between these two effects must be performed: moderate values of $\ell^{\star}$ and small enough high-frequency asymptotic gains. An interesting future direction is to cast a nonlinear optimization problem for optimally choosing $r$. An additional degree of freedom that is not exploited in this work is the possibility of a co-design of the gain $K_{n}$ and the filter parameters, as done, e.g., in [28] but for the filter structure proposed in this paper. We regard these ideas as interesting future research directions.
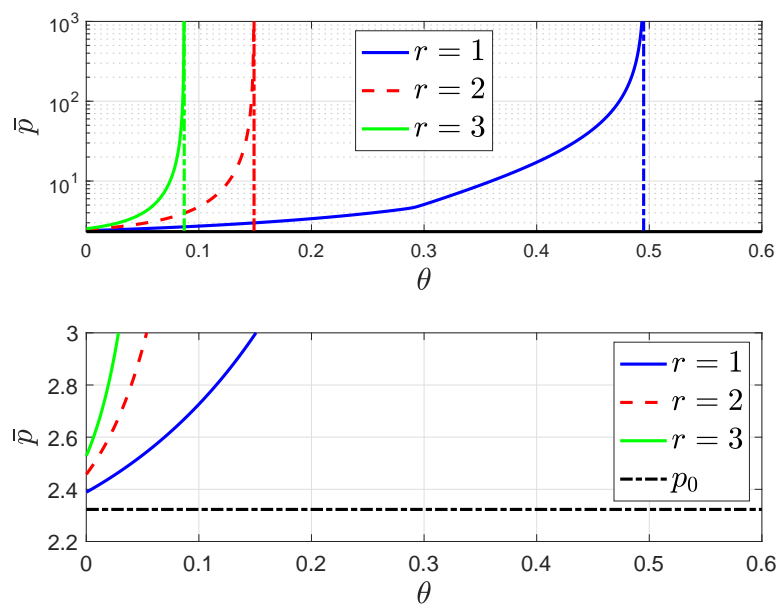

Figure 4: Value of the maximum eigenvalue $\bar{p}$ of $P(16)$ as a function of $\theta$, for $r=1,2,3$. Top figure, in logarithmic scale: Vertical asymptotes for $\theta^{\star}$ given in Table 1. Bottom figure, in linear scale: $\bar{p}_{0}$ computed from (18).

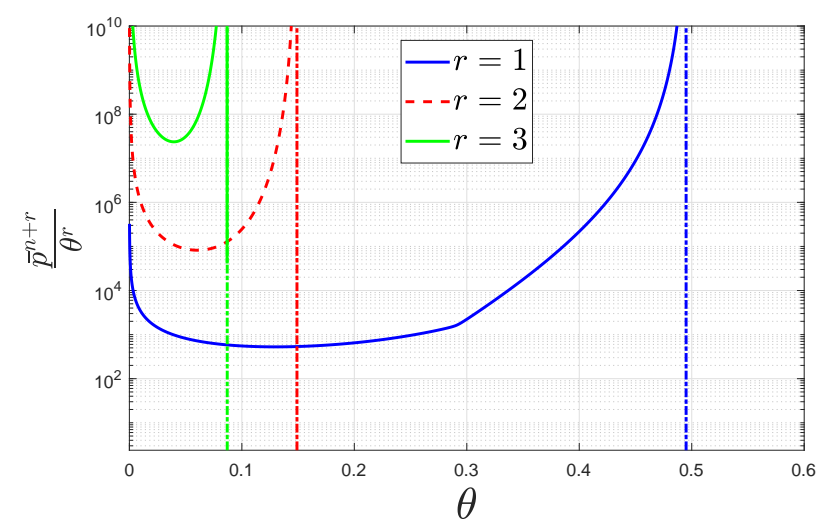

Figure 5: Value of ratio (40), with $\bar{p}$ computed as the maximum eigenvalue of $P$ in (16), for $r=1,2,3$. The figure is in logarithmic scale. The values of $\theta$ corresponding to the minimum of each curve are reported in Table 1.

\section{Conclusion}

We considered high-gain observers for nonlinear plants in observability canonical form and well characterized the simple, yet effective, idea of inserting a linear filter with a parametric relative degree in the output injection channel. The proposed filter is suitably parametrized by the high-gain parameter of the 


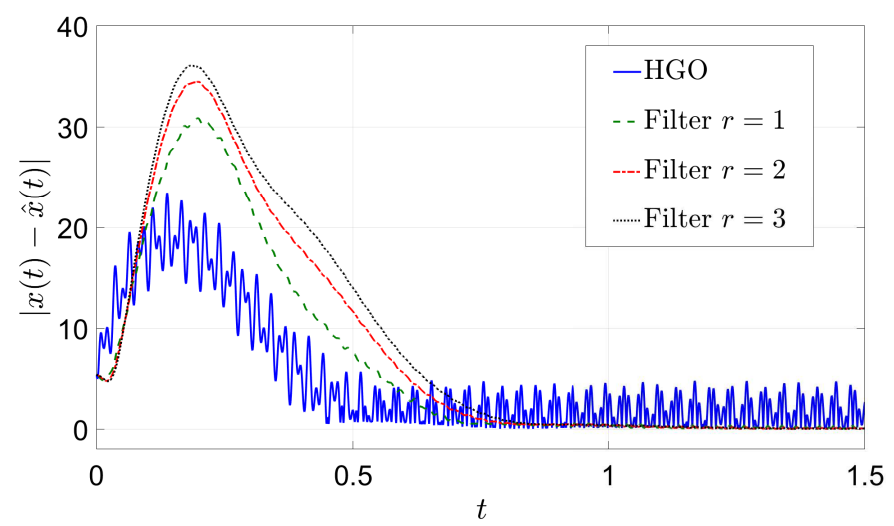

Figure 6: Norm of the estimation error $|x(t)-\hat{x}(t)|$ of system (41) for a high-gain observer (4), (5), and a filtered high-gain observer (4), (10) with $r=1,2,3$.

observer and an additional parameter whose tuning rule is simple and intuitive. For the proposed architecture, we first proved a parametric exponential finite-gain bound on the estimation error from the measurement noise. Then we characterized the effect of high-frequency noise using a parametric exosystem approach, showing that the high-frequency gain depends in a desirable and convenient way on the filter parameters. The theoretical results about the trends of the amplitude of the estimation error induced by high-frequency noise are confirmed by numerical simulation studies. Since the proposed filtering technique is based on a suitable time-scale separation, as a future work it would be interesting to use a similar idea for different observer design techniques.

\section{References}

[1] D. Astolfi, A. Alessandri, and L. Zaccarian. Stubborn ISS redesign for nonlinear high-gain observers. IFAC-PapersOnLine, vol. 50(1), pp. 1542215427, 2017.

[2] D. Astolfi, A. Alessandri, and L. Zaccarian. Stubborn and Dead-Zone Redesign for Nonlinear Observers and Filters. IEEE Transactions on Automatic Control, 2021.

[3] D. Astolfi, M. Jungers, L. Zaccarian. Output injection filtering redesign in high-gain observers. 2018 European Control Conference (ECC), pp. 19571962, 2018

[4] D. Astolfi and L. Marconi. A high-gain nonlinear observer with limited gain power, IEEE Transactions on Automatic Control. vol. 60 (11), pp. 3059-3064, 2015.

[5] D. Astolfi, L. Marconi, L. Praly and A. R. Teel. Sensitivity to highfrequency measurement noise of nonlinear high-gain observers. 10th Nonlinear Control System Symposium (NOLCOS), pp. 862-866, 2016.

[6] D. Astolfi, L. Marconi, L. Praly and A. R. Teel. Peaking-free low-power high-gain observers. Automatica, vol. 98, pp. 169-179, 2018.

[7] D. Astolfi, L. Marconi and A. R. Teel. Peaking-free low-power high-gain observers for nonlinear systems. 2016 European Control Conference (ECC), pp. 1424-1429, 2016.

[8] A.N. Atassi and H. K. Khalil. A separation principle for the stabilization of a class of nonlinear systems. IEEE Transactions on Automatic Control, vol. 44 (9), pp. 1672-1687, 1999.

[9] K.K. Busawon and P. Kabore. Disturbance attenuation using proportional integral observers. International Journal of control, vol. 74(6), pp. 618-627, 2001.

[10] K.K. Busawon, S. Danaher and P. Kabore. Observer design using lowpass filtered outputs. Proceedings of the 40th IEEE Conference on Decision and Control, vol. 4, pp. 3878-3879, 2001.

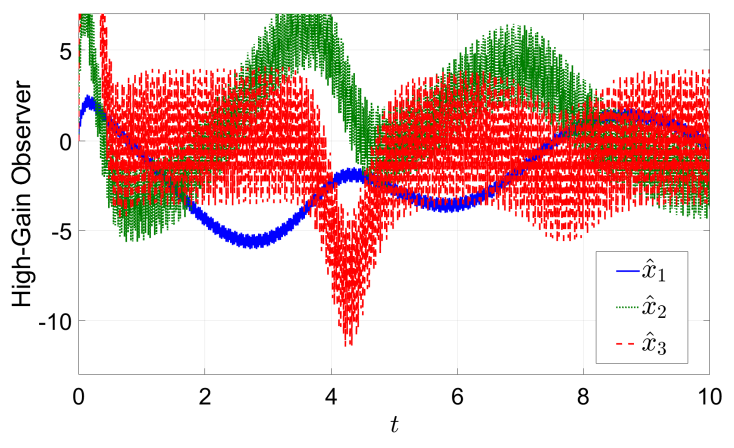

Figure 7: Components of the estimate $\hat{x}(t)$ of a high-gain observer (4), (5) for system (41) in the presence of measurement noise (8), (42).

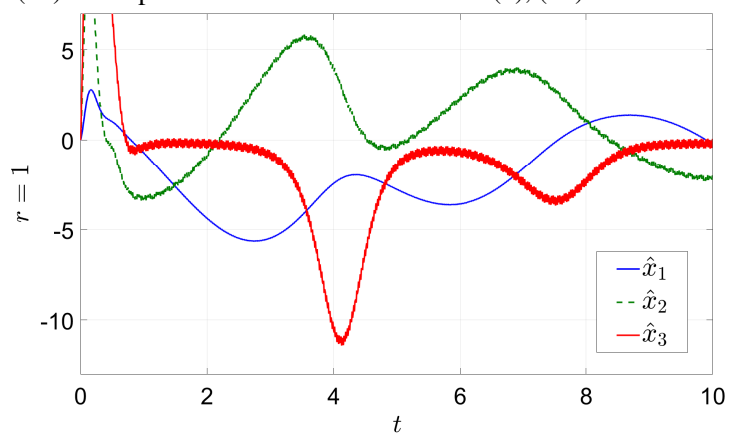

Figure 8: Components of the estimate $\hat{x}(t)$ of a filtered high-gain observer (4), (10) of dimension $r=1$ for system (41) in the presence of measurement noise (8), (42).

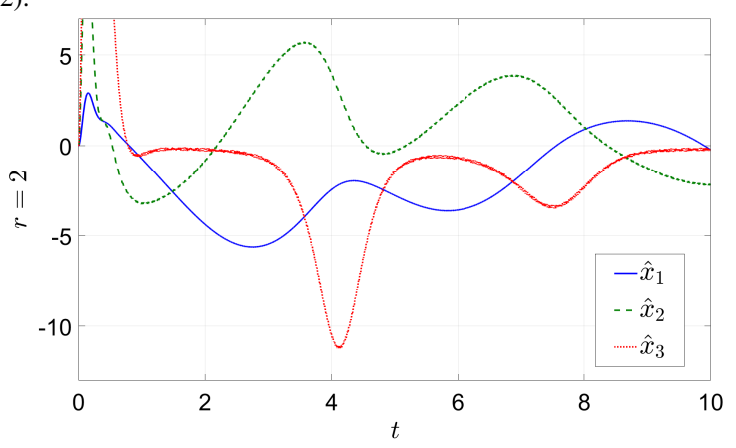

Figure 9: Components of the estimate $\hat{x}(t)$ of a filtered high-gain observer (4), (10) of dimension $r=2$ for system (41) in the presence of measurement noise (8), (42).

[11] K. W. Chang. Singular perturbations of a general boundary value problem. SIAM J. Mathemat. Anal., vol. 3, pp. 520-526, 1972.

[12] K.E. Chlouverakis and J. C. Sprott. Chaotic hyperjerk systems, Chaos, Solitons \& Fractals, vol. 28, pp. 739-746, 2006.

[13] M. Cocetti, S. Tarbouriech, and L. Zaccarian. High-gain dead-zone observers for linear and nonlinear plants. IEEE Control Systems Letters, vol. 3(2), pp. 356-361, 2018.

[14] J. P. Gauthier and I. Kupka. Deterministic observation theory and applications. Cambridge university press, 2001.

[15] A. Isidori and C. I. Byrnes. Steady-state behaviors in nonlinear systems with an application to robust disturbance rejection. Annual Reviews in Control, vol. 32(1), pp. 1-16, 2008.

[16] M. Jungers and C. Oară. Solving parameterized Non-symmetric Algebraic Riccati Equations: A Matrix Sign Function Approach. Journal of Control Engineering and Applied Informatics (CEAI), vol. 21 (2), pp. 3-10, 2019.

[17] M. Karow and D. Kressner. On a perturbation bound for invariant sub- 
spaces of matrices. SIAM J. Matrix Anal. Appl., 35(2), pp. 599-618, 2014.

[18] H. K. Khalil. Cascade High-Gain Observers in Output Feedback Control. Automatica, vol. 80, pp. 110-118, 2017.

[19] H. K. Khalil and L. Praly. High-gain observers in nonlinear feedback control. International Journal of Robust and Nonlinear Control, vol. 24 (6), pp. 993-1015, 2014.

[20] H. K. Khalil and S. Priess. Analysis of the use of low-pass filters with high-gain observers. IFAC-PapersOnLine, 49(18), 488-492, 2016.

[21] P. Kokotović, H. K. Khalil, J. O'Reilly. Singular Perturbation Methods in Control: Analysis and Design. Society for Industrial and Applied Mathematics, 1999.

[22] W. H. Kwon, Y. S. Moon, S. C. Ahn. Bounds in algebraic Riccati and Lyapunov equations: a survey and some new results. International Journal of Control, vol. 64 (3), 377-389, 1996.

[23] A. Levant, M. Livne. Robust exact filtering differentiators. European Journal of Control, 2019.

[24] L. Menini, C. Possieri and A. Tornambe. Observers for Linear Systems by the Time Integrals and Moving Average of the Output. IEEE Transactions on Automatic Control, vol. 64(12), pp. 4859-4874, 2019.

[25] A. M. Samoilenko. Elements of the Mathematical Theory of MultiFrequency Oscillations. Springer, 1991.

[26] A. R. Teel. Further variants of the Astolfi/Marconi high-gain observer 2016 American Control Conference (ACC), pp. 993-998, 2016.

[27] C. Tréangle, M. Farza, M. M'Saad. A simple filtered high gain observer for a class of uncertain nonlinear systems. 18th International Conference on Sciences and Techniques of Automatic Control and Computer Engineering (STA), pp. 396-401, 2017.

[28] C. Tréangle, M. Farza, M. M'Saad. Filtered high gain observer for a class of uncertain nonlinear systems with sampled outputs. Automatica, vol. 101, pp. 197-206, 2019. 